\title{
Permanence for a Discrete Ratio-Dependent Predator-Prey System with Holling Type III Functional Response and Feedback Controls
}

\author{
Jiangbin Chen ${ }^{1}$ and Shengbin $\mathbf{Y u}^{2}$ \\ ${ }^{1}$ Zhicheng College, Fuzhou University, Fuzhou, Fujian 350002, China \\ ${ }^{2}$ Sunshine College, Fuzhou University, Fuzhou, Fujian 350015, China \\ Correspondence should be addressed to Jiangbin Chen; chen-jiangbin@163.com
}

Received 6 October 2013; Accepted 2 December 2013

Academic Editor: Candace M. Kent

Copyright (C) 2013 J. Chen and S. Yu. This is an open access article distributed under the Creative Commons Attribution License, which permits unrestricted use, distribution, and reproduction in any medium, provided the original work is properly cited.

A new set of sufficient conditions for the permanence of a ratio-dependent predator-prey system with Holling type III functional response and feedback controls are obtained. The result shows that feedback control variables have no influence on the persistent property of the system, thus improving and supplementing the main result of Yang (2008).

\section{Introduction}

The aim of this paper is to investigate the permanent property of the following discrete ratio-dependent predator-prey system with Holling type III and feedback controls:

$$
\begin{aligned}
& \begin{aligned}
x(n+1) & \\
=x(n) \exp \{ & a(n)-b(n) x(n) \\
& \left.\quad-\frac{c(n) x(n) y(n)}{x^{2}(n)+m^{2}(n) y^{2}(n)}-e_{1}(n) u_{1}(n)\right\},
\end{aligned} \\
& \begin{aligned}
y(n+1) \\
=y(n) \exp \left\{\begin{array}{l}
-d(n) \\
\end{array}\right. \\
\left.\quad+\frac{f(n) x^{2}(n)}{x^{2}(n)+m^{2}(n) y^{2}(n)}-e_{2}(n) u_{2}(n)\right\}, \\
\Delta u_{1}(n)=-\eta_{1}(n) u_{1}(n)+q_{1}(n) x(n), \\
\Delta u_{2}(n)=-\eta_{2}(n) u_{2}(n)+q_{2}(n) y(n),
\end{aligned}
\end{aligned}
$$

where $x(t)$ and $y(t)$ are the densities of the prey population and predator population at time $t$, respectively, for $i=1$, $2,\{a(n)\},\{b(n)\},\{c(n)\},\{d(n)\},\{f(n)\},\{m(n)\},\left\{e_{i}(n)\right\},\left\{\eta_{i}(n)\right\}$, and $q_{i}(n)$ are all bounded nonnegative sequences such that

$$
\begin{gathered}
0<a^{L} \leq a(n) \leq a^{U}, \quad 0<b^{L} \leq b(n) \leq b^{U}, \\
0<c^{L} \leq c(n) \leq c^{U}, \\
0<d^{L} \leq d(n) \leq d^{U}, \quad 0<f^{L} \leq f(n) \leq f^{U}, \\
0<m^{L} \leq m(n) \leq m^{U}, \\
0<e_{i}^{L} \leq e_{i}(n) \leq e_{i}^{U}, \quad 0<\eta_{i}^{L} \leq \eta_{i}(n) \leq \eta_{i}^{U}<1, \\
0<q_{i}^{L} \leq q_{i}(n) \leq q_{i}^{U} .
\end{gathered}
$$

Here, for any bounded sequence $\{a(n)\}, a^{L}=\inf _{n \in N}\{a(n)\}$, $a^{U}=\sup _{n \in N}\{a(n)\}$.

By the biological meaning, we will focus our discussion on the positive solutions of system (1). So, we consider (1) together with the following initial conditions:

$$
x(0)>0, \quad y(0)>0,
$$$$
u_{i}(0)>0, \quad i=1,2 .
$$ 
It is not difficult to see that the solutions of (1)-(3) are well defined and satisfy

$$
\begin{gathered}
x(n)>0, \quad y(n)>0, \quad u_{i}(n)>0, \\
i=1,2, \quad \text { for } n \in N .
\end{gathered}
$$

Recently, Yang [1] proposed and studied the permanence of system (1). Set

$$
\begin{gathered}
M_{1}=\frac{\exp \left\{a^{U}-1\right\}}{b^{L}}, \quad M_{2}=\frac{f^{U} M_{1} \exp \left\{f^{U}-d^{L}\right\}}{2 d^{L} m^{L}}, \\
W_{1}=\frac{q_{1}^{U} M_{1}}{\eta_{1}^{L}}, \quad W_{2}=\frac{q_{2}^{U} M_{2}}{\eta_{2}^{L}} .
\end{gathered}
$$

Using the comparison theorem of difference equation, Yang obtained the following result.

Theorem A (see [1]). Assume that

$$
\begin{gathered}
a^{L}-\frac{c^{U}}{2 m^{L}}-e_{1}^{U} W_{1}>0, \\
f^{L}-d^{U}-e_{2}^{U} W_{2}>0,
\end{gathered}
$$

hold; then system (1) is permanent.

Theorem A shows that feedback control variables play important roles in the persistent property of the system (1). But the question is whether or not the feedback control variables have influence on the permanence of the system. On the other hand, as was pointed out by Fan and Wang [2], "if we use the method of comparison theorem, then the additional condition, in some extent, is necessary. But for the system itself, this condition may not necessary.[sic]" In [2], by establishing a new difference inequality, Fan and Wang showed that feedback control has no influence on the permanence of a single species discrete model. Their success motivated us to consider the persistent property of system (1). Indeed, in this paper, we will apply the analysis technique of Fan and Wang [2] to establish sufficient conditions, which is independent of feedback control variables, to ensure the permanence of the system. We finally obtain the following main results.

Theorem B. Assume that

$$
\begin{gathered}
a^{L}-\frac{c^{U}}{2 m^{L}}>0, \\
f^{L}-d^{U}>0,
\end{gathered}
$$

hold; then system (1) is permanent.

Comparing with Theorem $\mathrm{A}$, it is easy to see that $\left(H_{i}\right)$ in Theorem $\mathrm{B}$ are weaker than $\left(A_{i}\right)$ in Theorem $\mathrm{A}(i=$ $1,2)$ and feedback control variables have no influence on the permanent property of system (1), so our results improve the main results in [1]. For more works on this direction, one could refer to [3-10] and the references cited therein.
The remaining part of this paper is organized as follows. In Section 2, we will introduce several lemmas. The permanence of system (1) is then studied in Section 3. In Section 4, a suitable example together with its numerical simulations shows the feasibility of our results.

\section{Preliminaries}

In this section, we will introduce several useful lemmas.

Lemma 1 (see [11]). Assume that $\{x(n)\}$ satisfies

$$
x(n+1) \geq x(n) \exp \{a(n)-b(n) x(n)\}, \quad n \geq N_{0},
$$

$\limsup _{n \rightarrow+\infty} x(n) \leq x^{*}$ and $x\left(N_{0}\right)>0$, where $a(n)$ and $b(n)$ are nonnegative sequences bounded above and below by positive constants and $N_{0} \in N$. Then

$$
\liminf _{n \rightarrow+\infty} x(n) \geq \min \left\{\frac{a^{L}}{b^{U}} \exp \left\{a^{L}-b^{U} x^{*}\right\}, \frac{a^{L}}{b^{U}}\right\} .
$$

Lemma 2 (see [2]). Assume that $A>0$ and $y(0)>0$. Suppose that

$$
y(n+1) \leq A y(n)+B(n), \quad n=1,2, \ldots
$$

Then, for any integer $k \leq n$,

$$
y(n) \leq A^{k} y(n-k)+\sum_{i=0}^{k-1} A^{i} B(n-i-1)
$$

In particular, if $A<1$ and $B$ is bounded above with respect to $M$, then

$$
\limsup _{n \rightarrow+\infty} y(n) \leq \frac{M}{1-A} \text {. }
$$

Lemma 3 (see [2]). Assume that $A>0$ and $y(0)>0$. Suppose that

$$
y(n+1) \geq A y(n)+B(n), \quad n=1,2, \ldots
$$

Then, for any integer $k \leq n$,

$$
y(n) \geq A^{k} y(n-k)+\sum_{i=0}^{k-1} A^{i} B(n-i-1) .
$$

In particular, if $A<1$ and $B$ is bounded above with respect to $m^{*}$, then

$$
\liminf _{n \rightarrow+\infty} y(n) \geq \frac{m^{*}}{1-A}
$$

\section{Permanence}

In this section, we detail the proof of our main result by several lemmas. The following lemma is a direct conclusion of [1]. 
Lemma 4. There exist two positive constants $M_{1}$ and $W_{1}$ such that

$$
\begin{array}{cc}
\limsup _{n \rightarrow+\infty} x(n) \leq M_{1}, & \underset{n \rightarrow+\infty}{\limsup } y(n) \leq M_{2}, \\
\limsup _{n \rightarrow+\infty} u_{1}(n) \leq W_{1}, & \limsup _{n \rightarrow+\infty} u_{2}(n) \leq W_{2},
\end{array}
$$

where $M_{i}$ and $W_{i}(i=1,2)$ are defined in (5).

Lemma 5. Assume

$$
a^{L}-\frac{c^{U}}{2 m^{L}}>0
$$

holds then there exist two positive constants $m_{1}$ and $w_{1}$ such that

$$
\liminf _{n \rightarrow+\infty} x(n) \geq m_{1}, \quad \liminf _{n \rightarrow+\infty} u_{1}(n) \geq w_{1},
$$

where $m_{1}$ and $w_{1}$ are defined in the proof.

Proof. According to Lemma 4, for any $\varepsilon>0$ small enough, there exists enough large $N_{1}>0$, such that, for $n \geq N_{1}$,

$$
x(n) \leq M_{1}+\varepsilon, \quad u_{1}(n) \leq W_{1}+\varepsilon .
$$

Thus, it follows from (16) and the first equation of system (1) that

$$
\begin{aligned}
x(n+1) \geq & x(n) \\
& \times \exp \left\{a^{L}-b^{U}\left(M_{1}+\varepsilon\right)-\frac{c^{U}}{2 m^{L}}-e_{1}^{U}\left(W_{1}+\varepsilon\right)\right\} \\
\geq & x(n) \exp \left\{-b^{U}\left(M_{1}+\varepsilon\right)-\frac{c^{U}}{2 m^{L}}-e_{1}^{U}\left(W_{1}+\varepsilon\right)\right\} \\
\triangleq & x(n) \exp \left\{D_{1 \varepsilon}\right\},
\end{aligned}
$$

for $n \geq N_{1}$, where $D_{1 \varepsilon}=-b^{U}\left(M_{1}+\varepsilon\right)-\left(c^{U} / 2 m^{L}\right)-e_{1}^{U}\left(W_{1}+\varepsilon\right)<$ 0 . For any integer $k \leq n$, it follows from (17) that

$$
\prod_{j=n-k}^{n-1} \frac{x(j+1)}{x(j)} \geq \prod_{j=n-k}^{n-1} \exp \left\{D_{1 \varepsilon}\right\}=\exp \left\{D_{1 \varepsilon} k\right\}
$$

Thus

$$
x(n-k) \leq x(n) \exp \left\{-D_{1 \varepsilon} k\right\} .
$$

From the third equation of system (1), we have

$$
\begin{aligned}
u_{1}(n+1) & =\left(1-\eta_{1}(n)\right) u_{1}(n)+q_{1}(n) x(n) \\
& \leq\left(1-\eta_{1}^{L}\right) u_{1}(n)+q_{1}^{U} x(n) \\
& \stackrel{\Delta}{=} A_{1} u_{1}(n)+B_{1}(n),
\end{aligned}
$$

where $A_{1}=1-\eta_{1}^{L}$ and $B_{1}(n)=q_{1}^{U} x(n)$. Then, for any $k \leq n$, according to Lemma 2, (19), and (20)

$$
\begin{aligned}
u_{1}(n) & \leq A_{1}^{k} u_{1}(n-k)+\sum_{i=0}^{k-1} A_{1}^{i} B_{1}(n-i-1) \\
& =A_{1}^{k} u_{1}(n-k)+\sum_{i=0}^{k-1} A_{1}^{i} q_{1}^{U} x(n-i-1) \\
& \leq A_{1}^{k} u_{1}(n-k)+q_{1}^{U} x(n) \sum_{i=0}^{k-1} A_{1}^{i} \exp \left\{-D_{1 \varepsilon}(i+1)\right\}
\end{aligned}
$$

Note that $0<\eta_{1}^{L}<1$; hence $0<A_{1}<1$. Therefore,

$$
0 \leq A_{1}^{k} u_{1}(n-k) \leq A_{1}^{k}\left(W_{1}+\varepsilon\right) \longrightarrow 0, \quad \text { as } k \longrightarrow \infty .
$$

Then, there exists a positive integer $N_{2}>N_{1}$ such that, for any positive solution of system $(1), e_{1}^{U} A_{1}^{N_{2}}\left(W_{1}+\varepsilon\right)<(1 / 2)\left(a^{L}-\right.$ $\left.c^{U} / 2 m^{L}\right)$ for all $n \geq N_{2}$. In fact, we could choose $N_{2}=$ $\ln P_{1} / \ln A_{1}+1$, where $P_{1}=\left(2 m^{L} a^{L}-c^{U}\right) / 4 e_{1}^{U} m^{L}\left(W_{1}+\varepsilon\right)$. Fixing $N_{2}$, for $n \geq N_{1}+N_{2}$, we get

$$
\begin{aligned}
u_{1}(n) \leq & A_{1}^{N_{2}} u_{1}\left(n-N_{2}\right) \\
& +q_{1}^{U} x(n) \sum_{i=0}^{N_{2}-1} A_{1}^{i} \exp \left\{-D_{1 \varepsilon}(i+1)\right\} \\
\leq & A_{1}^{N_{2}}\left(W_{1}+\varepsilon\right)+q_{1}^{U} x(n) \sum_{i=0}^{N_{2}-1} A_{1}^{i} \exp \left\{-D_{1 \varepsilon}(i+1)\right\} \\
\triangleq & A_{1}^{N_{2}}\left(W_{1}+\varepsilon\right)+G_{1 \varepsilon} x(n),
\end{aligned}
$$

where $G_{1 \varepsilon}=q_{1}^{U} \sum_{i=0}^{N_{2}-1} A_{1}^{i} \exp \left\{-D_{1 \varepsilon}(i+1)\right\}$.

Substituting (23) into the first equation of system (1), we can get

$$
\begin{aligned}
& x(n+1) \\
& \geq x(n) \exp \left\{a^{L}-b^{U} x(n)-\frac{c^{U}}{2 m^{L}}-e_{1}^{U} u_{1}(n)\right\} \\
& \geq x(n) \exp \left\{a^{L}-b^{U} x(n)-\frac{c^{U}}{2 m^{L}}\right. \\
& =x(n) \exp \left\{a_{1}^{U}\left(A_{1}^{N_{2}}\left(W_{1}+\frac{c^{U}}{2 m^{L}}-e_{1}^{U} A_{1}^{N_{2}}\left(W_{1}+\varepsilon\right) \text { G } G_{1 \varepsilon} x(n)\right)\right\}\right. \\
& \left.\quad-\left(b^{U}+e_{1}^{U} G_{1 \varepsilon}\right) x(n)\right\} \\
& \geq x(n) \exp \left\{\frac{1}{2}\left(a^{L}-\frac{c^{U}}{2 m^{L}}\right)-\left(b^{U}+e_{1}^{U} G_{1 \varepsilon}\right) x(n)\right\} \\
& \triangleq x(n) \exp \left\{E_{1}-E_{2 \varepsilon} x(n)\right\},
\end{aligned}
$$

where $E_{1}=(1 / 2)\left(a^{L}-c^{U} / 2 m^{L}\right)$ and $E_{2 \varepsilon}=b^{U}+e_{1}^{U} G_{1 \varepsilon}$. 
By applying Lemma 1 to (24), it immediately follows that

$$
\liminf _{n \rightarrow+\infty} x(n) \geq \min \left\{\frac{E_{1}}{E_{2 \varepsilon}} \exp \left\{E_{1}-E_{2 \varepsilon} M_{1}\right\}, \frac{E_{1}}{E_{2 \varepsilon}}\right\} .
$$

Setting $\varepsilon \rightarrow 0$ in the above inequality, we obtain

$$
\liminf _{n \rightarrow+\infty} x(n) \geq \min \left\{\frac{E_{1}}{E_{2}} \exp \left\{E_{1}-E_{2} M_{1}\right\}, \frac{E_{1}}{E_{2}}\right\} \triangleq m_{1} .
$$

It follows from (26) that there exists large enough $N_{3} \geq N_{1}+$ $\mathrm{N}_{2}$ such that

$$
x(n) \geq \frac{m_{1}}{2}, \quad \forall n \geq N_{3} .
$$

This together with the third equation of system (1) leads to

$$
\Delta u_{1}(n) \geq-\eta_{1}(n) u_{1}(n)+\frac{m_{1} q_{1}(n)}{2}, \quad \forall n \geq N_{3} .
$$

Hence,

$$
u_{1}(n+1) \geq\left(1-\eta_{1}^{U}\right) u_{1}(n)+\frac{m_{1} q_{1}^{L}}{2}, \quad \forall n \geq N_{3}
$$

By applying Lemma 3, it follows from (29) that

$$
\liminf _{n \rightarrow+\infty} u_{1}(n) \geq \frac{m_{1} q_{1}^{L}}{2 \eta_{1}^{U}} \triangleq w_{1} .
$$

This completes the proof of Lemma 5.

Lemma 6. Assume that

$$
f^{L}-d^{U}>0
$$

holds; then there exist two positive constants $m_{2}$ and $w_{2}$ such that

$$
\liminf _{n \rightarrow+\infty} y(n) \geq m_{2}, \quad \liminf _{n \rightarrow+\infty} u_{2}(n) \geq w_{2}
$$

where $m_{2}$ and $w_{2}$ are defined in the proof.

Proof. According to Lemmas 4 and 5, for any $\varepsilon>0$ small enough, there exists enough large $N_{4}>0$, such that, for $n \geq$ $N_{4}$

$$
y(n) \leq M_{1}+\varepsilon, \quad x(n) \geq m_{1}-\varepsilon, \quad u_{2}(n) \leq W_{2}+\varepsilon .
$$

Thus, it follows from (32) and the second equation of system (1) that

$$
\begin{aligned}
& y(n+1) \\
& =y(n) \exp \left\{-d(n)+f(n)-\frac{f(n) m^{2}(n) y^{2}(n)}{x^{2}(n)+m^{2}(n) y^{2}(n)}\right. \\
& \left.\quad-e_{2}(n) u_{2}(n)\right\} \\
& \geq y(n) \exp \left\{-d^{U}+f^{L}-\frac{f(n) m(n) y(n)}{2 x(n)}-e_{2}^{U}\left(W_{2}+\varepsilon\right)\right\} \\
& \geq y(n) \exp \left\{-d^{U}+f^{L}-\frac{f^{U} m^{U}\left(M_{1}+\varepsilon\right)}{2\left(m_{1}-\varepsilon\right)}-e_{2}^{U}\left(W_{2}+\varepsilon\right)\right\} \\
& \geq y(n) \exp \left\{-d^{U}-\frac{f^{U} m^{U}\left(M_{1}+\varepsilon\right)}{2\left(m_{1}-\varepsilon\right)}-e_{2}^{U}\left(W_{2}+\varepsilon\right)\right\} \\
& \triangleq y(n) \exp \left\{D_{2 \varepsilon}\right\},
\end{aligned}
$$

for $n \geq N_{4}$, where $D_{2 \varepsilon}=-d^{U}-f^{U} m^{U}\left(M_{1}+\varepsilon\right) / 2\left(m_{1}-\varepsilon\right)-$ $e_{2}^{U}\left(W_{2}+\varepsilon\right)<0$. For any integer $k \leq n$, it follows from (33) that

$$
\prod_{j=n-k}^{n-1} \frac{y(j+1)}{y(j)} \geq \prod_{j=n-k}^{n-1} \exp \left\{D_{2 \varepsilon}\right\}=\exp \left\{D_{2 \varepsilon} k\right\} .
$$

Thus

$$
y(n-k) \leq y(n) \exp \left\{-D_{2 \varepsilon} k\right\} .
$$

From the fourth equation of system (1), we can get

$$
\begin{aligned}
u_{2}(n+1) & =\left(1-\eta_{2}(n)\right) u_{2}(n)+q_{2}(n) y(n) \\
& \leq\left(1-\eta_{2}^{L}\right) u_{2}(n)+q_{2}^{U} y(n) \\
& \stackrel{\Delta}{=} A_{2} u_{2}(n)+B_{2}(n),
\end{aligned}
$$

where $A_{2}=1-\eta_{2}^{L}$ and $B_{2}(n)=q_{2}^{U} y(n)$. Then, for any $k \leq n$, according to Lemma 2, (35) and (36), that

$$
\begin{aligned}
u_{2}(n) & \leq A_{2}^{k} u_{2}(n-k)+\sum_{i=0}^{k-1} A_{2}^{i} B_{2}(n-i-1) \\
& =A_{2}^{k} u_{2}(n-k)+\sum_{i=0}^{k-1} A_{2}^{i} q_{2}^{U} y(n-i-1) \\
& \leq A_{2}^{k} u_{2}(n-k)+q_{2}^{U} y(n) \sum_{i=0}^{k-1} A_{2}^{i} \exp \left\{-D_{2 \varepsilon}(i+1)\right\} .
\end{aligned}
$$

Note that $0<\eta_{2}^{L}<1$; hence $0<A_{2}<1$. Therefore,

$$
0 \leq A_{2}^{k} u_{2}(n-k) \leq A_{2}^{k}\left(W_{2}+\varepsilon\right) \longrightarrow 0, \quad \text { as } k \longrightarrow \infty .
$$


Then, there exists a positive integer $N_{5}>N_{4}$ such that, for any positive solution of system (1), $e_{2}^{U} A_{2}^{N_{5}}\left(W_{2}+\varepsilon\right)<(1 / 2)\left(f^{L}-d^{U}\right)$ for all $n \geq N_{5}$. In fact, we could choose $N_{5}=\ln P_{2} / \ln A_{2}+1$, where $P_{2}=\left(f^{L}-d^{U}\right) / 2 e_{2}^{U}\left(W_{2}+\varepsilon\right)$. Fixing $N_{5}$, for $n \geq N_{4}+N_{5}$, we get

$$
\begin{aligned}
u_{2}(n) & \leq A_{2}^{N_{5}} u_{2}\left(n-N_{5}\right)+q_{2}^{U} y(n) \sum_{i=0}^{N_{5}-1} A_{2}^{i} \exp \left\{-D_{2 \varepsilon}(i+1)\right\} \\
& \leq A_{2}^{N_{5}}\left(W_{2}+\varepsilon\right)+q_{2}^{U} y(n) \sum_{i=0}^{N_{5}-1} A_{2}^{i} \exp \left\{-D_{2 \varepsilon}(i+1)\right\} \\
& \triangleq A_{2}^{N_{5}}\left(W_{2}+\varepsilon\right)+G_{2 \varepsilon} y(n),
\end{aligned}
$$

where $G_{2 \varepsilon}=q_{2}^{U} \sum_{i=0}^{N_{5}-1} A_{2}^{i} \exp \left\{-D_{2 \varepsilon}(i+1)\right\}$.

Substituting (39) into the second equation of system (1), we can get

$$
\begin{aligned}
& y(n+1) \geq y(n) \\
& \times \exp \left\{-d^{U}+f^{L}-\frac{f(n) m(n) y(n)}{2 x(n)}-e_{2}^{U} u_{2}(n)\right\} \\
& \geq y(n) \exp \left\{-d^{U}+f^{L}-\frac{f^{U} m^{U} y(n)}{2\left(m_{1}-\varepsilon\right)}\right.\left.-e_{2}^{U}\left(A_{2}^{N_{5}}\left(W_{2}+\varepsilon\right)+G_{2 \varepsilon} y(n)\right)\right\} \\
&=y(n) \exp \left\{-d^{U}+f^{L}-e_{2}^{U} A_{2}^{N_{5}}\left(W_{2}+\varepsilon\right)\right.\left.-\left(\frac{f^{U} m^{U}}{2\left(m_{1}-\varepsilon\right)}+e_{2}^{U} G_{2 \varepsilon}\right) y(n)\right\} \\
& \geq y(n) \exp \left\{\frac{1}{2}\left(-d^{U}+f^{L}\right)\right. \\
&\left.-\left(\frac{f^{U} m^{U}}{2\left(m_{1}-\varepsilon\right)}+e_{2}^{U} G_{2 \varepsilon}\right) y(n)\right\} \\
& \triangleq y(n) \exp \left\{E_{3}-E_{4 \varepsilon} y(n)\right\},
\end{aligned}
$$

for all $n \geq N_{5}$, where $E_{3}=(1 / 2)\left(-d^{U}+f^{L}\right)$ and $E_{4 \varepsilon}=$ $f^{U} m^{U} / 2\left(m_{1}-\varepsilon\right)+e_{2}^{U} G_{2 \varepsilon}$.

By applying Lemma 1 to (40), it immediately follows that

$\liminf _{n \rightarrow+\infty} y(n) \geq \min \left\{\frac{E_{3}}{E_{4 \varepsilon}} \exp \left\{E_{3}-E_{4 \varepsilon} M_{2}\right\}, \frac{E_{3}}{E_{4 \varepsilon}}\right\}$.

Setting $\varepsilon \rightarrow 0$ in the above inequality, we obtain

$$
\liminf _{n \rightarrow+\infty} y(n) \geq \min \left\{\frac{E_{3}}{E_{4}} \exp \left\{E_{3}-E_{4} M_{1}\right\}, \frac{E_{3}}{E_{4}}\right\} \triangleq m_{2} .
$$

It follows from (42) that there exists large enough $N_{6} \geq N_{4}+$ $\mathrm{N}_{5}$ such that

$$
y(n) \geq \frac{m_{2}}{2}, \quad \forall n \geq N_{6} .
$$

This together with the fourth equation of system (1) leads to

$$
\Delta u_{2}(n) \geq-\eta_{2}(n) u_{2}(n)+\frac{m_{2} q_{2}(n)}{2}, \quad \forall n \geq N_{6} .
$$

Hence,

$$
u_{2}(n+1) \geq\left(1-\eta_{2}^{U}\right) u_{2}(n)+\frac{m_{2} q_{2}^{L}}{2}, \quad \forall n \geq N_{6} .
$$

By applying Lemma 3, it follows from (45) that

$$
\liminf _{n \rightarrow+\infty} u_{2}(n) \geq \frac{m_{2} q_{2}^{L}}{2 \eta_{2}^{U}} \stackrel{\Delta}{=} w_{2} .
$$

This completes the proof of Lemma 6 .

Lemmas 4-6 show that the conclusion of Theorem B holds.

\section{Example and Numerical Simulation}

Consider the following system:

$$
\begin{aligned}
& x(n+1) \\
& =x(n) \exp \{1.32+0.32 \sin (\sqrt{17} n) \\
& -(5.3+0.2 \sin (n)) x(n) \\
& -\frac{(0.75+0.25 \sin (\sqrt{7} n)) x(n) y(n)}{x^{2}(n)+(0.8+0.2 \cos (\sqrt{11} n))^{2} y^{2}(n)} \\
& \left.-(2+0.1 \cos (\sqrt{13} n)) u_{1}(n)\right\} \text {, } \\
& y(n+1) \\
& =y(n) \exp \{-(0.125+0.025 \cos (\sqrt{5} n)) \\
& +\frac{(0.25+0.05 \sin (\sqrt{3} n)) x^{2}(n)}{x^{2}(n)+(0.8+0.2 \cos (\sqrt{11} n))^{2} y^{2}(n)} \\
& \left.-(1.5+0.5 \sin (n)) u_{2}(n)\right\} \text {. }
\end{aligned}
$$

$$
\begin{aligned}
\Delta u_{1}(n)= & -(0.09+0.01 \sin (\sqrt{7} n)) u_{1}(n) \\
& +(0.3+0.2 \cos (n)) x(n), \\
\Delta u_{2}(n)= & -(0.06+0.04 \cos (\sqrt{11} n)) u_{2}(n) \\
& +(0.1+0.05 \sin (n)) y(n) .
\end{aligned}
$$



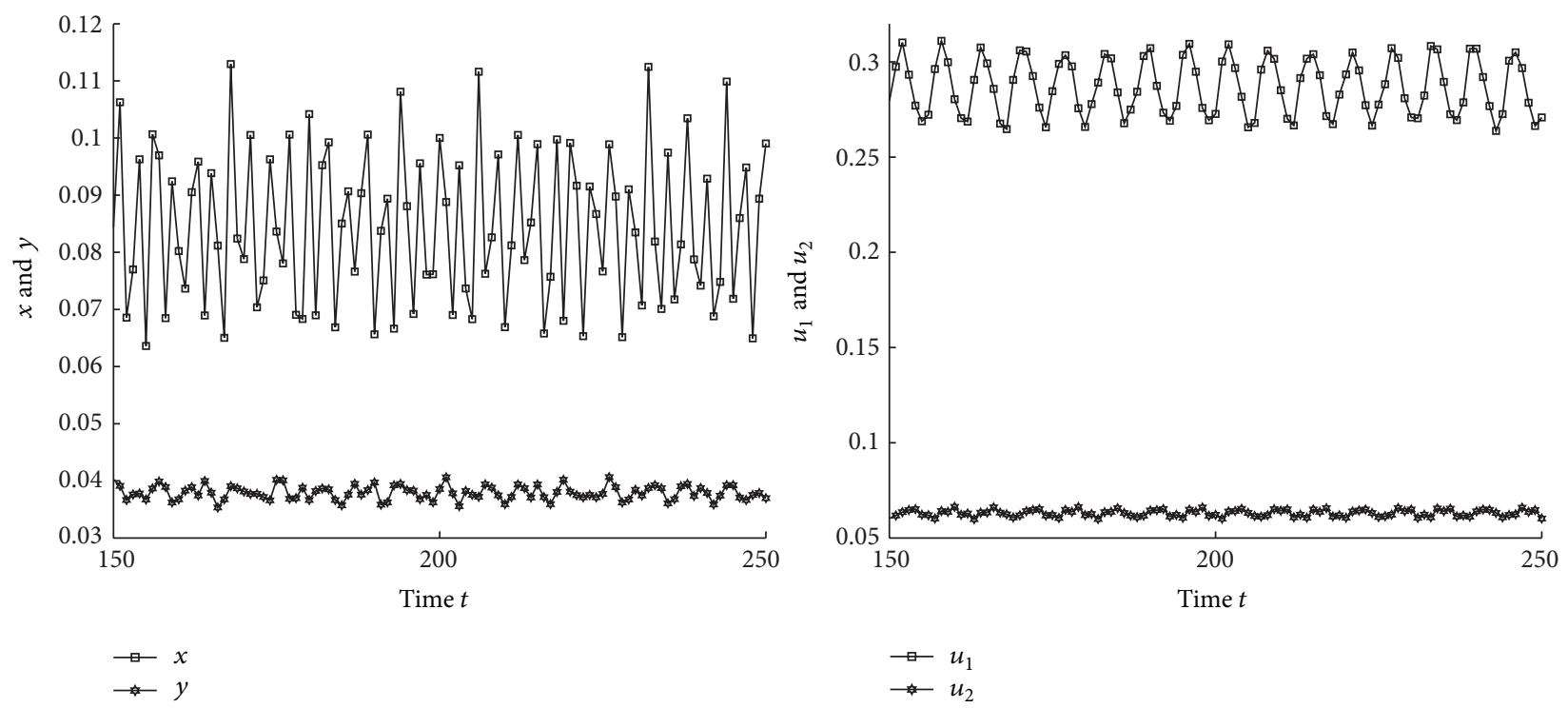

FIGURE 1: Dynamic behavior of the system (47) with the initial condition $\left(x(0), y(0), u_{1}(0), u_{2}(0)\right)=(0.2,0.4,0.1,0.14)^{T}$ and $(0.4,0.5,0.21$, $0.3)^{T}$, respectively.

In this case, we have

$$
\begin{gathered}
a^{L}-\frac{c^{U}}{2 m^{L}} \approx 0.1667>0, \\
f^{L}-d^{U}=0.05>0 .
\end{gathered}
$$

Equation (48) means that all conditions of Theorem B are satisfied in system (47). Thus, the system (47) is permanent. Our numerical simulation supports our result (see Figure 1). However,

$$
a^{L}-\frac{c^{U}}{2 m^{L}}-e_{1}^{U} W_{1} \approx-4.7139<0,
$$

that is to say, $\left(A_{1}\right)$ does not hold and we could not obtain the result of the permanence from Theorem A. Thus our results improve the main results in [1].

\section{Acknowledgment}

This research is supported by the Foundation of Fujian Education Bureau (JA11294 and JA13365).

\section{References}

[1] J. Yang, "Dynamics behaviors of a discrete ratio-dependent predator-prey system with holling type III functional response and feedback controls," Discrete Dynamics in Nature and Society, vol. 2008, Article ID 186539, 19 pages, 2008.

[2] Y. Fan and L. Wang, "Permanence for a discrete model with feedback control and delay," Discrete Dynamics in Nature and Society, vol. 2008, Article ID 945109, 8 pages, 2008.

[3] W. Yang and X. Li, "Feedback control has no influence on the persistent property of a single species discrete model with time delays," Mathematical and Computer Modelling, vol. 54, no. 9-10, pp. 2007-2013, 2011.
[4] L. Chen, X. Xie, and L. Chen, "Feedback control variables have no influence on the permanence of a discrete n-species cooperation system," Discrete Dynamics in Nature and Society, vol. 2009, Article ID 306425, 10 pages, 2009.

[5] Q. Su and N. Zhang, "Feedback control variables have no influence on the permanence of a discrete $\mathrm{n}$-species schoener competition system with time delays," Discrete Dynamics in Nature and Society, vol. 2010, Article ID 583203, 12 pages, 2010.

[6] G. Yu and H. Lu, "Permanence and almost periodic solutions of a discrete ratio-dependent Leslie system with time delays and feedback controls," Abstract and Applied Analysis, vol. 2012, Article ID 358594, 31 pages, 2012.

[7] L. Chen, L. Chen, and Z. Li, "Permanence of a delayed discrete mutualism model with feedback controls," Mathematical and Computer Modelling, vol. 50, no. 7-8, pp. 1083-1089, 2009.

[8] Z. Wang and Y. Li, "Almost periodic solutions of a discrete mutualism model with feedback controls," Discrete Dynamics in Nature and Society, vol. 2010, Article ID 286031, 18 pages, 2010.

[9] L. Wang and Y. Fan, "Permanence for a discrete Nicholson's blowflies model with feedback control and delay," International Journal of Biomathematics, vol. 1, no. 4, pp. 433-442, 2008.

[10] F. Chen, "Permanence of a discrete N-species cooperation system with time delays and feedback controls," Applied Mathematics and Computation, vol. 186, no. 1, pp. 23-29, 2007.

[11] F. Chen, "Permanence for the discrete mutualism model with time delays," Mathematical and Computer Modelling, vol. 47, no. 3-4, pp. 431-435, 2008. 


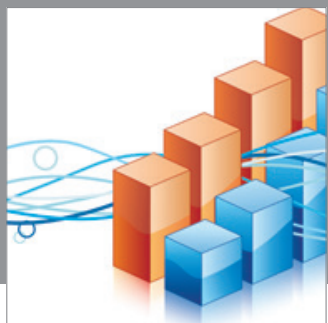

Advances in

Operations Research

mansans

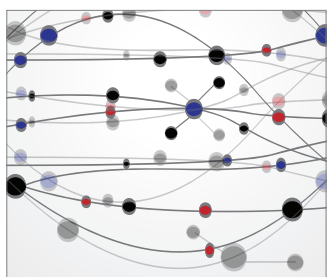

The Scientific World Journal
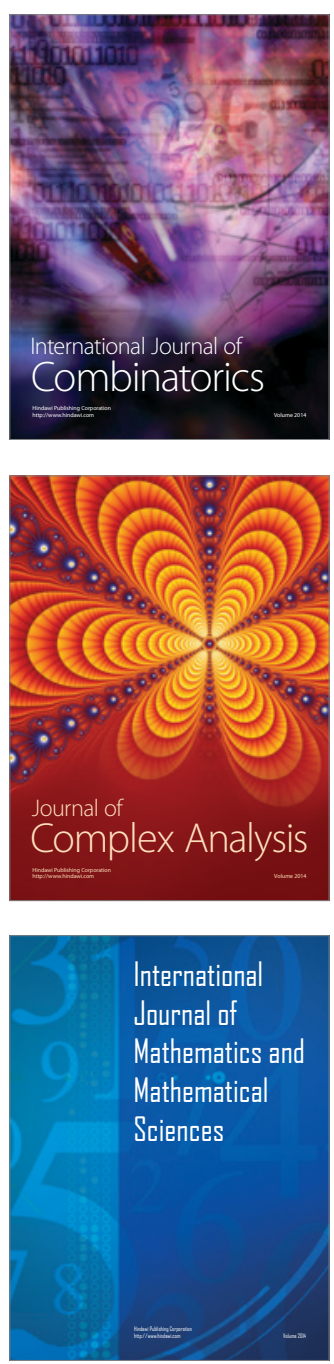
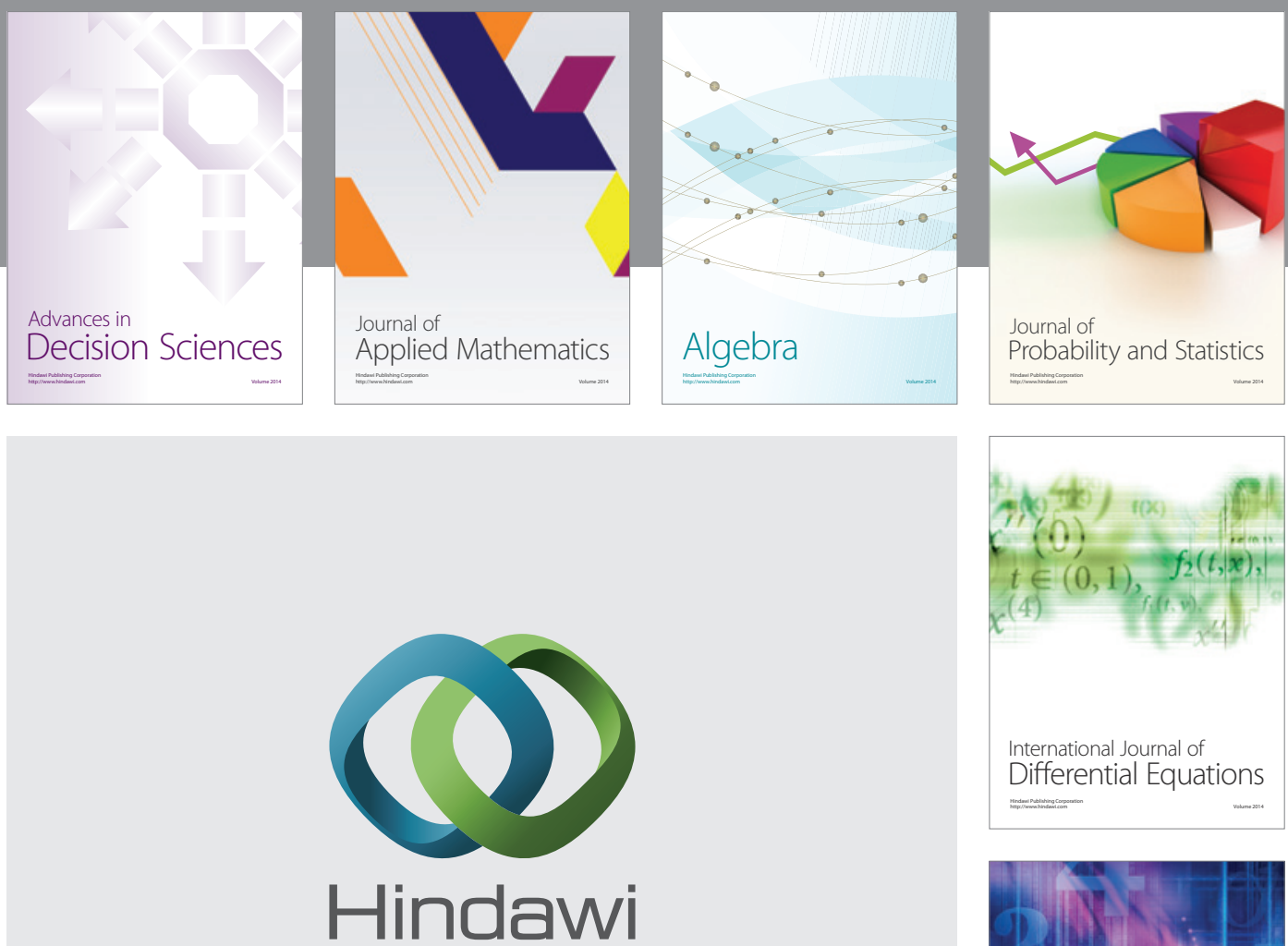

Submit your manuscripts at http://www.hindawi.com
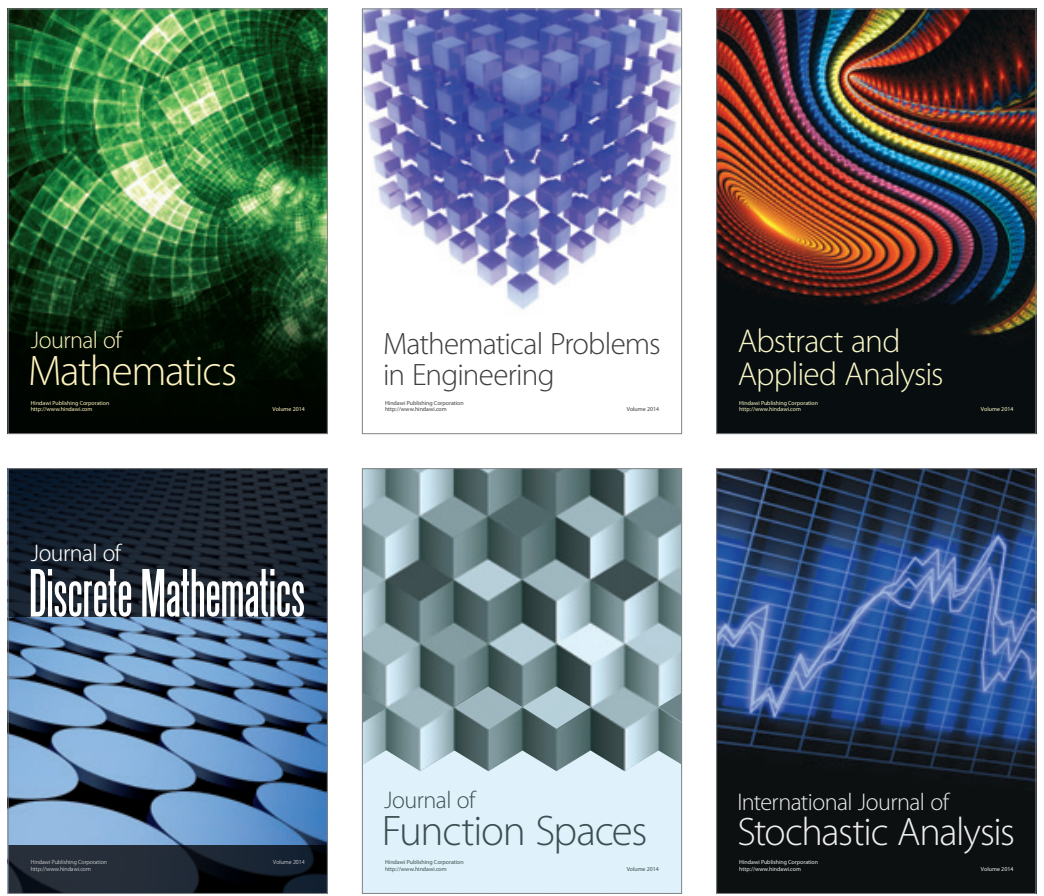

Journal of

Function Spaces

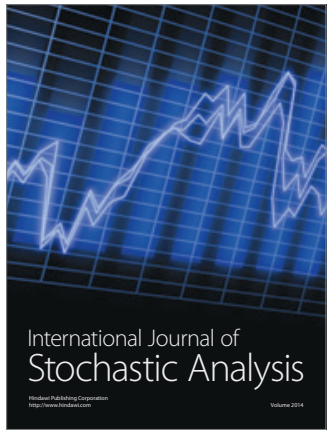

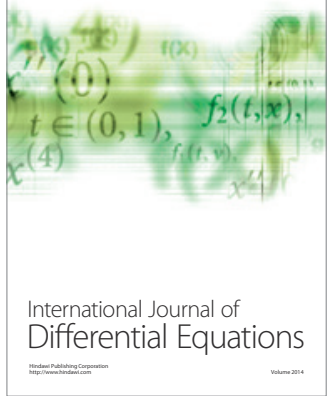
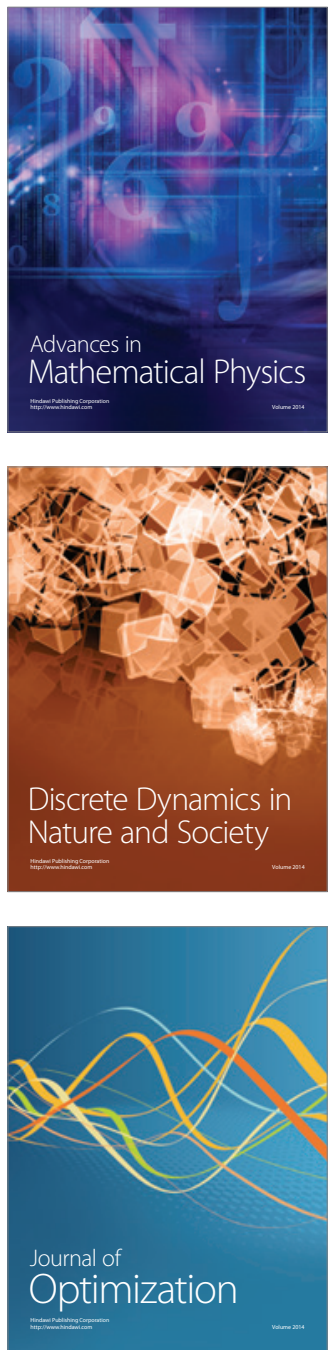